\title{
STRUCTURAL AND MAGNETIC PROPERTIES OF PURE AND B-SITE SUBSTITUTED BISMUTH FERRITE.
}

\author{
${ }^{1}$ R. Patel, 2P. S. Sawadh, \\ Dept. of Physics, B. D. College of Engineering, Sewagram, Wardha \\ E-mail : patel.rozina84@gmail.com
}

\begin{abstract}
:
Multiferroic $\mathrm{BiFeO}_{3}$ and $\mathrm{BiFe}_{1-\mathrm{x}}\left(\mathrm{Co}, \mathrm{Ni}_{\mathrm{x}} \mathrm{O}_{3}\right.$ materials were synthesized by autocombustion method using urea as a fuel. The crystal structure study was carried out using Xray diffraction (XRD). All synthesized samples crystallized in a rhombohedrally distorted $\mathrm{BiFeO}_{3}$ structure. With the increase in $\mathrm{Ni}$ doping concentration there is an increase in lattice parameters of the unit cell. However, the lattice parameters for Co doped samples are smaller than those for $\mathrm{BiFeO}_{3}$, which is due to the smaller ion diameter of $\mathrm{Co}^{3+}$ than that of $\mathrm{Fe}^{3+}$. Room temperature magnetic study showed that in pure $\mathrm{BiFeO}_{3}$, the magnetization varies nearly linearly with the applied magnetic field confirming that sample is antiferromagnetic having a Gtype magnetic structure. Compared with $\mathrm{BiFeO}_{3}$, the magnetic properties of doped samples were significantly enhanced.
\end{abstract}

Keywords: Multiferroics, magnetoelectric,auto-combustion, and $\mathrm{BiFeO}_{3}$

\section{Introduction :}

Multifunctional materials that combine a spontaneous magnetization with a ferroelectric polarization are of tremendous fundamental and practical interest ${ }^{[1-4]}$.Multiferroics are a class of materials with coupled electric, magnetic, and structural order parameters that yield simultaneous effects of ferroelectricity, ferromagnetism, and ferroelasticity in the same material[5].Magnetoelectricmultiferroics are the focus of muchexperimental and theoretical research [6-10]due to the potential to greatly impact the field of spinbased electronics (spintronics) including new forms of magnetic storage, logic, and memory[11,12].An ideal multiferroic would be aferroelectric ferromagnet that exhibits coupling between these orderparameters at room temperature. There has beengrowingrecent interest in the magnetic,ferroelectric, and piezoelectric properties of bismuth based perovskite-structure oxides ${ }^{[13-21]}$. In such 
materials, the formally $\mathrm{Bi}^{3+}$ ion occupies the perovskite $\mathrm{A}$ site, and its stereochemically active $6 \mathrm{~s}^{2}$ lone pair induces a symmetry-lowering structural distortion that can lead to ferroelectricity[22].The B-site substitutions are made by replacing the $\mathrm{Fe}^{3+}$ ions by other transition metal ions. Since the conduction band of $\mathrm{BiFeO}_{3}$ is related to the $\mathrm{d}$ orbital state of the $\mathrm{Fe}^{3+}$ ion, $\mathrm{B}$-site substitutions can have a strong influence on physical properties by changing the electronic structure near the Fermilevel.In this work we analyze the magnetic properties of Bi-based multiferroics in order to understand the chemical and structural factors which affect the magnetic ordering.

$\mathrm{BiFeO}_{3}$ is one such multifunctional material, which is ferroelectric $\left(\mathrm{T}_{\mathrm{C}} \sim 830^{\circ} \mathrm{C}\right)$ and the magnetic ordering is essentially G-type antiferromagnetic $\left(\mathrm{T}_{\mathrm{N}} \sim 370^{\circ} \mathrm{C}\right)$ with cycloidal spin magnetic ordering [23]. The necessary condition to manifest multiferroic properties in $\mathrm{BiFeO}_{3}$ is the suppression of spin cycloid, which is reported to be possible with application of high magnetic/electric fields, epitaxial strains, and chemical substitutions ${ }^{[24]}$. However, $\mathrm{BiFeO}_{3}$ usually exhibits a relatively high leakage current that is detrimental to ferroelectric performance and often makes $\mathrm{BiFeO}_{3}$ practically unusable ${ }^{25,26]}$.It is also difficult to derive weak ferromagnetism in pure $\mathrm{BiFeO}_{3}{ }^{[27]}$ whose spacemodulated spin structure may lead to a cancellation of the possible nonzero remnant magnetization permitted by the canted G-type antiferromagnetic order[28,29]. However to enhance the magnetic and ferroelectric properties of $\mathrm{BiFeO}_{3}$, chemical modification have been attempted with varying degrees of success ${ }^{[30-32]}$.

Inducing the lattice distortion is one way to enhance the magnetic properties of $\mathrm{BiFeO}_{3}$ by enhancing the canting of the antiferromagnetic arranged neighboring spins. Ion substitution is an efficient way to induce the lattice distortion to improve the magnetic properties. Fe-site ion substitution with magnetic ions is also very attracting, and enhanced ferromagnetism was reported[33]. In this paper, we present the structural and enhanced magnetic properties of $\mathrm{BiFeO}_{3}$ by $\mathrm{Co}$ and $\mathrm{Ni}$ substitution. 


\section{Experimental :}

The $\mathrm{BiFeO}_{3}, \mathrm{BiCo}_{\mathrm{x}} \mathrm{Fe}_{1-\mathrm{x}} \mathrm{O}_{3}(\mathrm{x}=0.5, \quad 0.8)$ and $\mathrm{BiNi}_{\mathrm{x}} \mathrm{Fe}_{1-\mathrm{x}} \mathrm{O}_{3}(\mathrm{x}=0.5,0.8)$ ceramics were prepared by auto-combustion synthesis. The chemicals $\mathrm{Bi}\left(\mathrm{NO}_{3}\right)_{3} .5 \mathrm{H}_{2} \mathrm{O}$ (99.9\% Merck), $\mathrm{Fe}\left(\mathrm{NO}_{3}\right)_{3} .9 \mathrm{H}_{2} \mathrm{O}(99.9 \% \mathrm{Merck}), \mathrm{Co}\left(\mathrm{NO}_{3}\right)_{2} \cdot 6 \mathrm{H}_{2} \mathrm{O}$ (99.9\% Merck), $\mathrm{Ni}\left(\mathrm{NO}_{3}\right)_{2} \cdot 6 \mathrm{H}_{2} \mathrm{O}$ and Urea(Merck) used were analytical reagent grade and used without further purification. Stoichiometric amount of metal nitrate (oxidizer) and fuel when ignited undergo self-propagating, gasproducing combustion reaction to yield voluminous metal oxide in less than 5 min. The as-synthesized powders were crushed and grinded in an agate mortar [34]. The oxidizer/fuel ratio was figured on the basis of oxidizing valencies of the metal nitrates and reducing valency of the fuel.

The synthesized powders were characterized by using various techniques. X-ray diffraction data was collected using a XPERT-PRO diffractometer with $\mathrm{Cu} \mathrm{ka}$ radiation $(\lambda=0.154056 \mathrm{~nm})$ at step of 0.02 in the range $2 \theta=20^{\circ}$ to $80^{\circ}$. The magnetic properties were measured by a vibrating sample magnetometer (Lakeshore VSM 7410) at room temperature.

\section{RESULTS AND DISCUSSION :}

\section{Structural Analysis}

Room temperature powder x-ray diffraction pattern of pure $\mathrm{BiFeO}_{3}$ and $\mathrm{BiNi}_{\mathrm{x}} \mathrm{Fe}_{1-\mathrm{x}} \mathrm{O}_{3}(\mathrm{x}=0.5,0.8)$ is presented in Figure[1-3]. All the peaks could be indexed in the rhombohedral distorted perovskite structure with space groupR3c.The planes (hkl) in the XRD pattern for $\mathrm{BiFeO}_{3}$ were matched by comparing them with the data of JCPD card of 86-1518. Using $2 \theta$ values from XRD plot and hkl values from the standard JCPD card the lattice parameters were found.The lattice parameters of pure $\mathrm{BiFeO}_{3}$ are relatively close to the reported literature and the same are calculated to be $a=b=5.5730^{\circ} \mathrm{A}$ and $\mathrm{c}=13.862^{\circ} \mathrm{A}$. The dopedsamples alsoexhibited similar rhombohedralperovskite structure. 


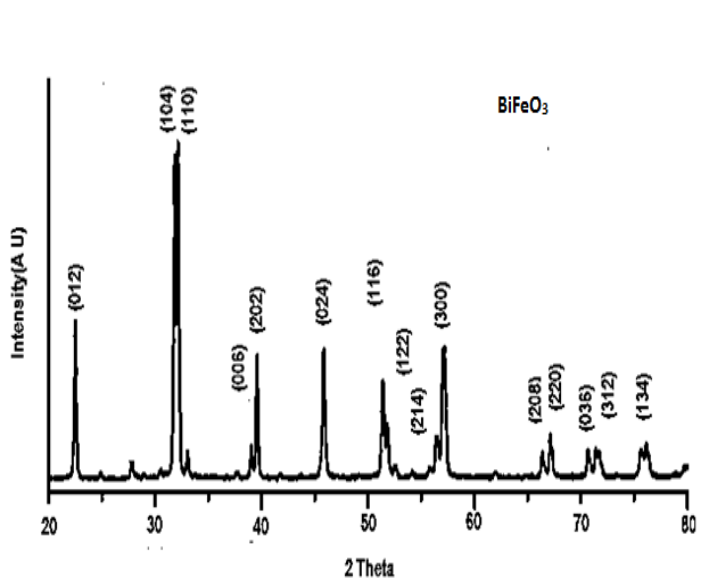

Fig [1]

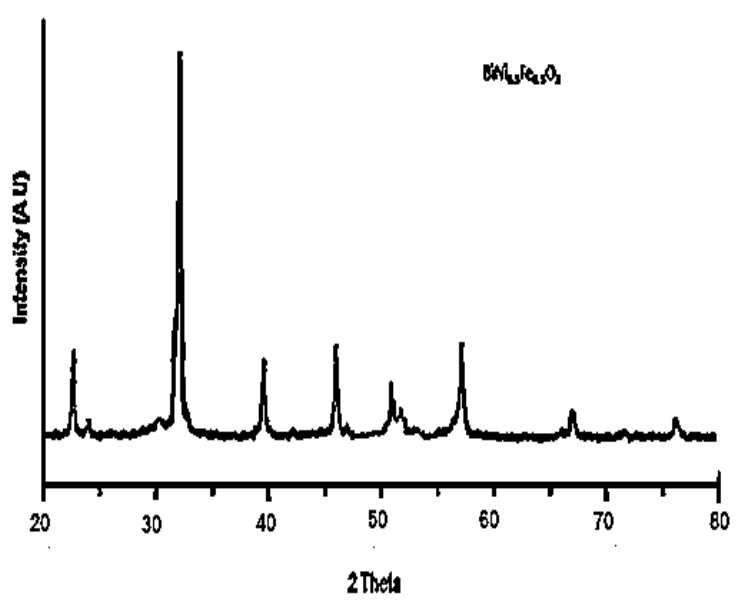

Fig [2]

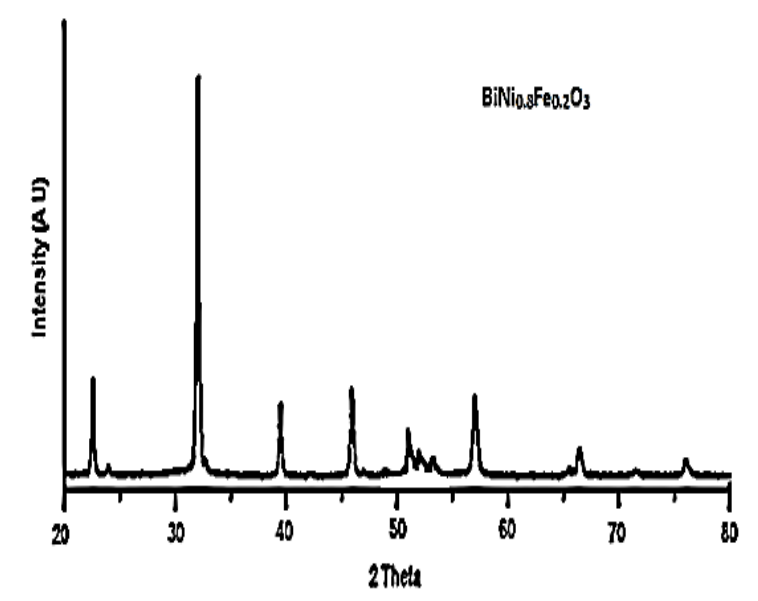

Fig [3]

The lattice parameters for $0.5 \mathrm{Ni}$ doped $\mathrm{BiFeO}_{3}$ are $\mathrm{a}=\mathrm{b}=5.5874^{\circ} \mathrm{A}$ and $\mathrm{c}=$ $13.879^{\circ} \mathrm{A}$, whereas for 0.8 , the values are $\mathrm{a}=\mathrm{b}=5.5976^{\circ} \mathrm{A}$ and $\mathrm{c}=13.89^{\circ} \mathrm{A}$.It can be seen clearly that with the increase in Ni doping concentration there is an increase in both, aandc parameters of the unit cell. This is as expected, since the ionic radius of $\mathrm{Ni}^{2+}$ is slightly larger than that of $\mathrm{Fe}^{3+}$. However, the lattice constant for Co doped samples are found to be smaller than thosefor $\mathrm{BiFeO}_{3}$, which is due to the smaller ion diameter of $\mathrm{Co}^{3+}$ than that of $\mathrm{Fe}^{3+}$. Hence, small structure contraction can be expected, giving riseto a slight canting of the rhombohedral unit cell[35]. Since there is a change in both, a and c parameters, the ratio c/a remains more or less unaffected, which is important for maintaining the ferroelectric properties in doped $\mathrm{BiFeO}_{3}$ samples. 


\section{Magnetic properties :}

The room temperature magnetic hysteresis loops are shown in figures[4]. In pure $\mathrm{BiFeO}_{3}$, the magnetization varies nearly linearly with the applied magnetic fieldconfirming that sample isantiferromagnetic having a G-type magnetic structure [36] and having a residual magnetic moment due to canted spin structure (weak ferromagnetic)..The magnetic parameters for $x=0.5 \mathrm{Ni}$ doped sample are $\mathrm{Ms}=0.278 \mathrm{emu} / \mathrm{g}$ and $\mathrm{Mr}=3.560 \mathrm{emu} / \mathrm{g}$ while saturation magnetizationMs $=0.687 \mathrm{emu} / \mathrm{g}$ and remanant magnetization $\mathrm{Mr}=1.1235$ emu/g, i.e. for $\mathrm{x}=0.8 \mathrm{Ni}$ doped sample are much larger than those of $\mathrm{BiFeO}_{3}$ prepared under similar conditions $\mathrm{Ms}=0.004 \mathrm{emu} / \mathrm{g}, \mathrm{Mr}=0.942 \mathrm{emu} / \mathrm{g}$ respectively.

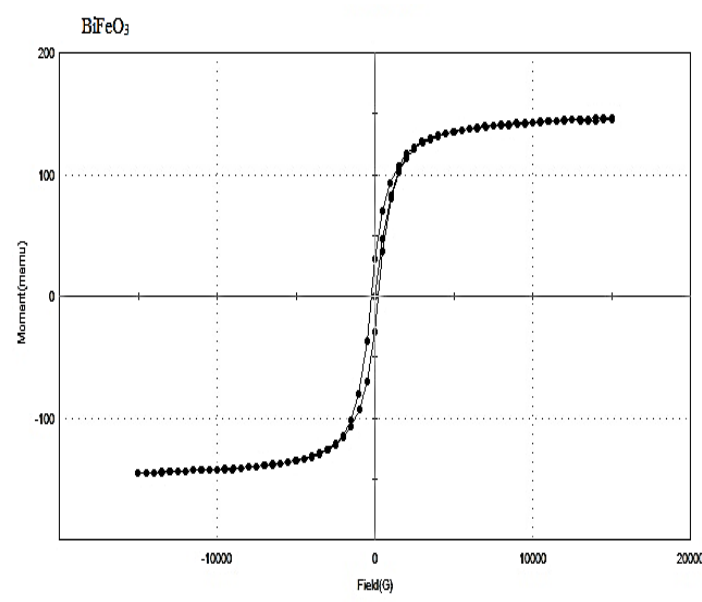

Fig [4]

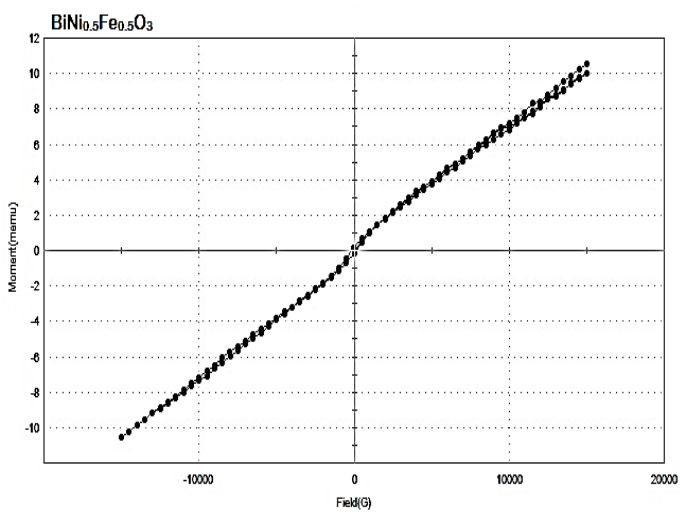

Fig [6]

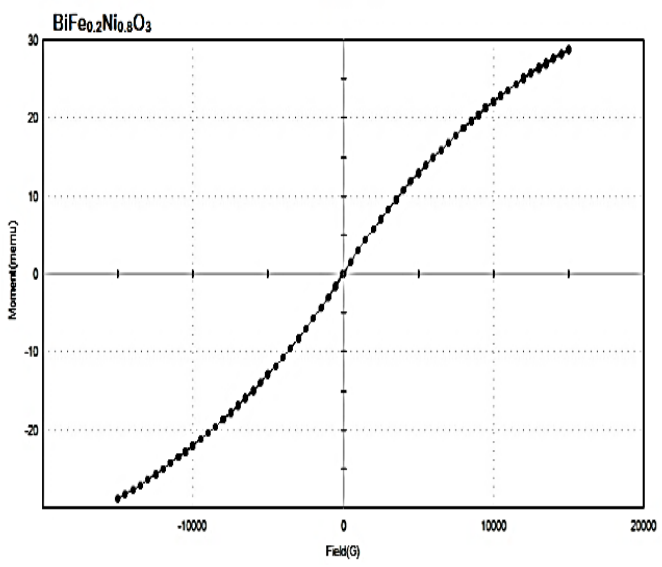

Fig [5] 
An enhanced moment was observed for the higher Co-doped samples as well. The enhancement of the magnetic parameters might berelated to the increased magnetoelectric coupling inside Co-doped $\mathrm{BiFeO}_{3}$.

\section{Conclusion:}

$\mathrm{BiFeO}_{3}$ and $\mathrm{BiFe}_{1-\mathrm{x}}(\mathrm{Co}, \mathrm{Ni})_{\mathrm{x}} \mathrm{O}_{3}$ materials were synthesized by autocombustion method. Co substitution at $\mathrm{Fe}$ site causes a structural distortion due to decrease in lattice parameter where as an increase in lattice parameter is observed for $\mathrm{Ni}$ substitution. Pure $\mathrm{BiFeO}_{3}$ exhibits antiferromagnetic behavior at room temperature. The $\mathrm{Co}$, Ni substitution has strengthened the magnetic properties of the bismuthferrite system. The spin structure in the magnetic ion substitution at Fesites in $\mathrm{BiFeO}_{3}$ needs further study to clearly understand the enhanced magnetic properties, and the room temperaturemultiferroic materials with enhanced properties might be realized.

\section{References:}

M. Fiebig, T. Lottermoser, D. Fr“ohlich, A. V. Goltsev, and R. V. Pisarev, (2002). Nature (London) 419, 818.

H. Zheng, J. Wang, S. E. Lofland, Z. Ma, L. Mohaddes-Ardabili, T. Zhao, L. Salamanca-Riba, S. R. Shinde, S. B. Ogale, and F. Bai, et al., (2004). Science 303, 661 .

M. Fiebig, V. Eremenko, and I. E. Chupis, (2004). eds., Proceedings of the 5th International workshop on magnetoelectric interaction phenomena in crystals (Kluwer, Dordrecht.

H. Schmid, (1994). Ferroelectrics 162, 317.

E. K. H. Salje, (1990). Phase Transitions in Ferroelectrics, Ferroelastic and Coelastic Crystals sCambridge University Press, Cambridge.

M. Fiebig, (2005). J. Phys. D: Appl. Phys 38, R123.

W. Eerenstein, N. D. Mathur, and J. F. Scott, (2006). Nature 442, 759. 
S.-W. Cheong and M. Mostovoy, (2007). Nature Mat. 6, 13.

R. Ramesh and N. A. Spaldin, (2007). Nature Mat. 6, 21.

H. Bea, M. Gajek, M. Bibes, and A. Barthelemy, (2008). J. Phys. C 20, 434221.

C. Binek and B. Doudin, (2005). J. Phys. C 17, L39.

Science Magazine features Science Watch on Multiferroics: Breakthrough of the Year 2007.

N. A. Hill and K. M. Rabe, (1999). Phys. Rev. B 59, 8759.

R. Seshadri and N. A. Hill, (2001). Chem. Mater. 13, 2892.

N. A. Hill, P. Baettig, and C. Daul, (2002). J. Phys. Chem. B 106, 3383.

J. Wang, J. B. Neaton, H. Zheng, V. Nagarajan, S. B. Ogale, B. Liu, D. Viehland, V. Vaithyanathan, D. G. Schlom, and U. V. Waghmare, et al., (2003). Science 299, 1719.

S. Niitaka, M. Azuma, M. Takano, E. Nishibori, M. Takata, and M. Sakata, (2004). Solid State Ionics 172, 557 (2004). 6 P. Baettig and N. A. Spaldin, Appl. Phys. Lett. 86, 012505(2005).

J. B. Neaton, C. Ederer, U. V. Waghmare, N. A. Spaldin, and K. M. Rabe, (2005). Phys. Rev. B 71, 14113.

C. Ederer and N. A. Spaldin, (2005). Phys. Rev. B 71, 60401.

F. Bai, J. Wang, M. Wuttig, J. Li, N. Wang, A. P. P.dn A. K. Zvezdin, L. E. Cross, and D. Viehland, (2005). Appl. Phys. Lett. 86, 32511.

P. Baettig, C. F. Schelle, R. LeSar, U. V. Waghmare, and N. A. Spaldin, (2005). Chem. Mater. 17, 1376.

R. Seshadri and N. A. Hill, (2001). Chem. Mater. 13, 2892.

I. Sosnowska, M. Lowenhaupt, W. I. F. David, and R. M. Ibberson, (1992). Physica B 180-181, 117.

A. D. Kadomtseva and Y. F. Popov, (2006). Phase Transitions 79, 1019. 
1W. Eerenstein, F. D. Morrison, J. Dho, M. G. Blamire, J. F. Scott, and N. D. Mathur, (2005). Science 307, 1203a.

G. W. Pabst, L. W. Martin, Y. H. Chu, and R. Ramesh, (2007). Appl. Phys. Lett. 90, 072902.

J. Dho, X. D. Qi, H. Kim, J. L. MacManus-Driscoll, and M. G. Blamire, (2006). Adv. Mater. 18, 1445.

B. Ruette, S. Zvyagin, A. P. Pyatakov, A. Bush, J. F. Li, V. I. Belotelov, A. K. Zvezdin, and D. Vieland, (2004). Phys. Rev. B 69, 064114.

F. Bai, J. Wang, M. Wuttig, J. F. Li, N. Wang, A. P. Pyatakov, A. K. Zvezdin, L. E. Cross, and D. Vieland, (2005). Appl. Phys. Lett. 86, 032511.

J. Wang, J. B. Neaton, H. Zheng, V. Nagarajan, S. B. Ogale, B. Liu, D. Viehland, V. Vaithyanathan, D. G. Schlom, U. V. Waghmare, N. A. Spaldin, K. M. Rabe, M. Wuttig, and R. Ramesh, (2003).Science, 299, 1719.

W. M. Zhu, and Z.-G. Ye, (2004). Ceram. Int., 30, 7, 1435.

W. Eerenstein, F. D. Morrison, J. Dho, M. G. Blamire, J. F. Scott, and N. D. Mathur, (2005). Science, 307, 1203a.)

P. Kharel, S. Talebi, B. Ramachandran, A. Dixit, V. M. Naik, M. B. Sahana, C. Sudakar, R. Naik, M. S. R. Rao, and G. Lawes, (2009). J. Phys.: Condens. Matter 21, 036001.

K C PATIL (1993). Bull. Mater. Sci., Vol. 16, NO. 6, December 1993, pp. 533541. Printed in India.

Y. WANG1, G. XU1, L. YANG2, Z. REN1, X. WEI1, W. WENG 1, P. DU1, G. SHEN1, G. HAN1 Materials Science-Poland, Vol. 27, No. 1, 2009 A.J. Jacobson, and B.E.F. Fender, (1975). J. Phys. C: Solid State Phys. 8, 844. 